Case Report

\title{
Atypical Lipomatous Tumor/Well-Differentiated Liposarcoma Developed in a Patient with Progressive Muscular Dystrophy: A Case Report and Review of the Literature
}

\author{
Ryo Miyagi, ${ }^{1,2}$ Toshihiko Nishisho, ${ }^{1,2}$ Shinjiro Takata, ${ }^{3}$ Yoshimitsu Shimatani, ${ }^{1}$ \\ Shunichi Toki, ${ }^{1,2}$ and Koichi Sairyo ${ }^{1,2}$ \\ ${ }^{1}$ Department of Orthopedics, Institute of Biomedical Sciences, Tokushima University Graduate School, Tokushima 770-8503, Japan \\ ${ }^{2}$ Department of Clinical Neuroscience, Institute of Biomedical Sciences, Tokushima University Graduate School, Tokushima, Japan \\ ${ }^{3}$ Department of Orthopedics, National Hospital Organization Tokushima Hospital, Tokushima, Japan
}

Correspondence should be addressed to Toshihiko Nishisho; tnishisho@tokushima-u.ac.jp

Received 20 February 2017; Accepted 13 April 2017; Published 29 May 2017

Academic Editor: Vivek Subbiah

Copyright ( 2017 Ryo Miyagi et al. This is an open access article distributed under the Creative Commons Attribution License, which permits unrestricted use, distribution, and reproduction in any medium, provided the original work is properly cited.

Background. Atypical lipomatous tumor/well-differentiated liposarcoma (ALT/WDLS) is an intermediate or locally aggressive form of adipocytic soft tissue sarcoma. Muscular dystrophy (MD) is characterized by progressive muscle atrophy and its replacement by adipose and fibrous tissue. Recently, some authors have reported that MD genes are related to neoplastic formation, but there have been no detailed clinical reports of ALT associated with MD. Case Presentation. A 73-year-old woman with a diagnosis of limb-girdle MD visited our department for recurrence of a huge tumor in her left thigh. She had undergone resection of a lipoma at the same site more than 20 years earlier. Imaging studies revealed a lipomatous tumor in her left thigh. We performed marginal resection including the adjacent muscles. Histological diagnosis was atypical lipomatous tumor. The postoperative course was uneventful, with no recurrence at 36 months of follow-up. Conclusion. We encountered a huge atypical tumor in a patient with MD. This is the first detailed report to describe an association between ALT and MD. We hypothesize that degenerative changes occurring in adipose tissue during muscle atrophy can cause lipomatous neoplasms and moreover that the mutation of MD-related genes may lead to the proliferation of tumor cells or to malignancy.

\section{Background}

Limb-girdle muscular dystrophy (LGMD) is characterized by progressive weakness of proximal muscles, of which one type involves the replacement of muscle by adipose and fibrous tissue [1-6]. Most muscular dystrophies (MDs) are caused by mutations in genes such as dystrophin, dysferlin, calpain, or LARGE like-glycosyltransferase [7-12].

Recently, these genes have been shown to be strongly related to neoplastic lesions. Schmidt et al. suggested that these genes might serve as tumor suppressors [12]. Several types of tumor, such as myeloma and rhabdomyosarcoma, have been reported to be related to MDs $[13,14]$. Here, we report the case of a 73-year-old patient with LGMD type 2B (LGMD2B) who developed an atypical lipomatous tumor (ALT), an intermediate or locally aggressive form of adipocytic tumor that is also referred to as well-differentiated liposarcoma (WDLS) [15].

\section{Case Presentation}

A 73-year-old woman with progressive weakness in the proximal muscles since age 50 was diagnosed with LGMD. Her parents were first cousins, and of her four younger siblings, three were diagnosed with LGMD. The patient underwent resection of a soft tissue tumor in the left thigh at age 49 . The pathological diagnosis was lipoma.

The tumor recurred in the left thigh at age 62. At the time of presentation to our department, the tumor had been present for 11 years and had gradually increased in size. 


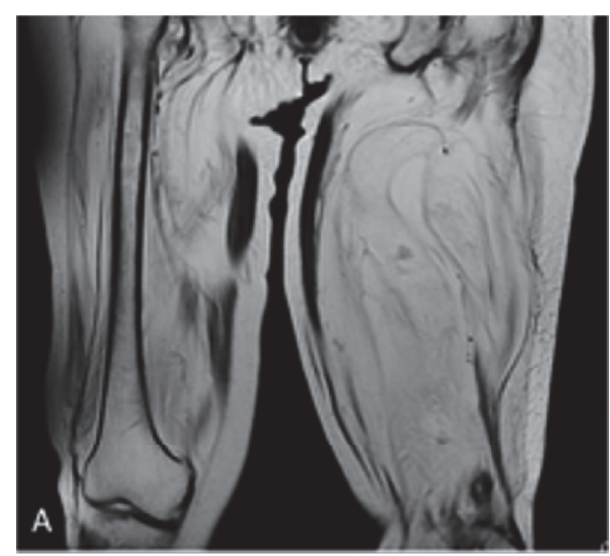

(a)

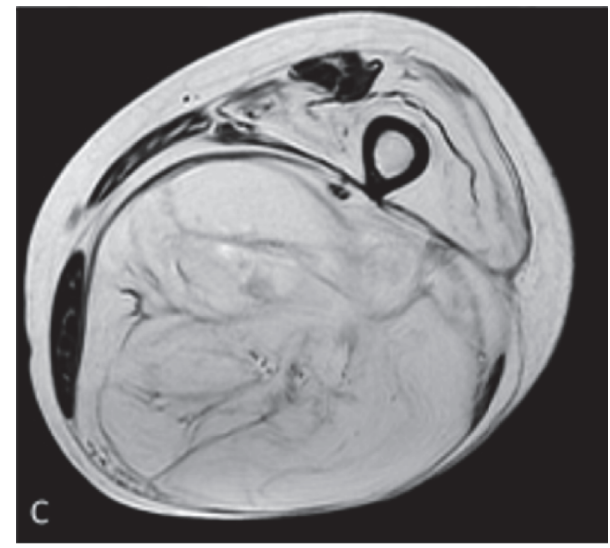

(c)

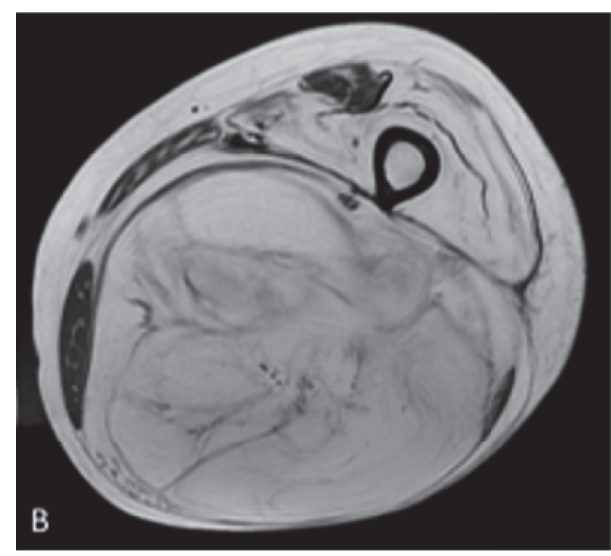

(b)

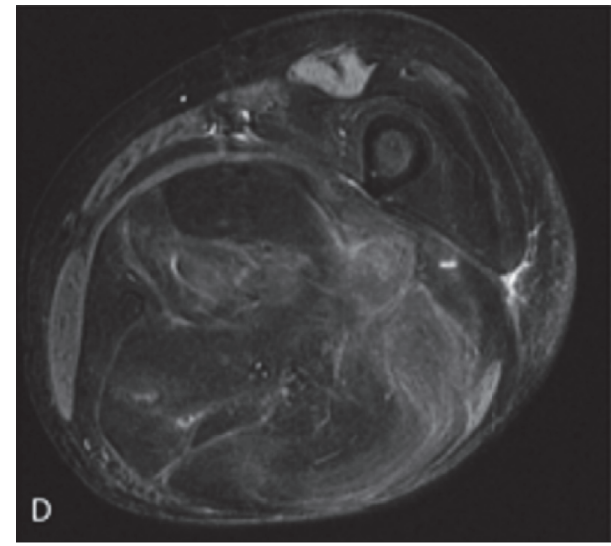

(d)

FIGURE 1: Magnetic resonance imaging revealed a tumor mass measuring $13 \times 15 \times 24 \mathrm{~cm}$ located in the left posterior thigh. Almost all the muscles, except the rectus femoris, sartorius, and gracilis muscles, show lipomatous changes. T1- and T2-weighted images (WIs) T2-WI, and short-TI inversion recovery (STIR) images show high intensity. Coronal T2-WI (a), T1-WI (axial view) (b), T2-WI (axial view) (c), and STIR images (axial view) (d).

Physical examination at first visit revealed an elastic hard mass measuring approximately $25 \mathrm{~cm}$ in width and $30 \mathrm{~cm}$ in length, located in the posterior left thigh. No tenderness, redness, or local warmth was noted. Laboratory examinations revealed that serum creatine kinase $(\mathrm{CK})$ was mildly elevated at $825 \mu \mathrm{IU} / \mathrm{L}$. Plain radiography showed a soft tissue mass shadow in the left thigh without calcification or periosteal reaction. Magnetic resonance imaging (MRI) revealed high signal intensity on T1- and T2-weighted images and heterogeneous hyperintensity on short-TI inversion recovery images (Figure 1). In addition, severe muscle atrophy and lipomatous changes were observed in both thighs. Open biopsy revealed no evidence of highly malignant cells. Therefore, we considered the tumor to be a lipoma or an atypical lipomatous tumor.

Surgical excision of the tumor was performed. On exploration, the tumor consisted of fat globules partially surrounded by a capsule and with poorly defined margins that was infiltrating different layers of the thigh muscles. We performed marginal resection including the semitendinosus muscle, semimembranosus muscle, and biceps femoris muscle (Figure 2). The resected tumor measured $15 \times 35 \times 20 \mathrm{~cm}$.
Pathological examination revealed that it contained mature adipocytes with cellular atypia (Figure 3). Immunohistochemical expression was positive for p16, MDM2, and CDK4. The final pathological diagnosis was atypical lipomatous tumor. The patient's postoperative course was uneventful, and there was no evidence of recurrence at 36 months postoperatively.

Since western blot analysis of muscle showed a deficit of dysferlin, the patient was finally diagnosed with LGMD2B.

\section{Discussion}

LGMD is characterized by progressive weakness of proximal muscles such as those of the hip or shoulder and was first proposed as a nosological entity by Walton and Nattrass in 1954 [1]. LGMD is classified into two main groups: autosomal dominant (LGMD type 1) and autosomal recessive (LGMD type 2). Autosomal recessive inheritance is more common. The symptoms of LGMD usually occur in the first and second decades of life; however, the onset, progression, and distribution of the weakness and wasting differ among the disease 


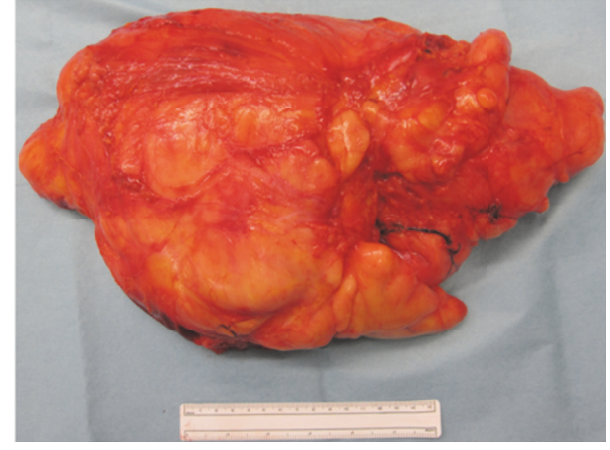

FIGURE 2: Macroscopic appearance of the resected tumor. The mass includes the semitendinosus muscle, semimembranosus muscle, and biceps femoris muscle.

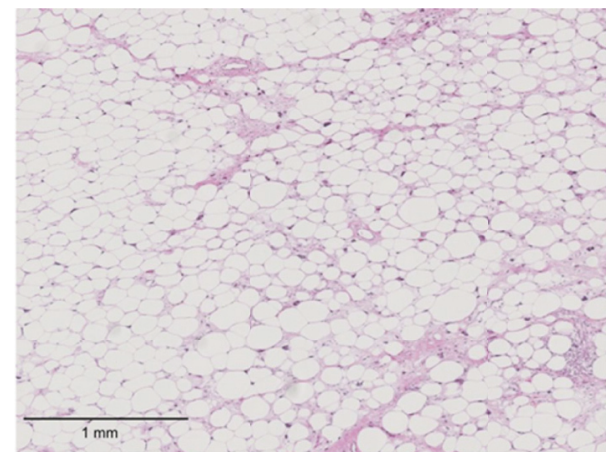

Figure 3: Pathological findings show mature adipocytes with cellular atypia and marked variations in size and shape. Hematoxylineosin staining, $\times 25$. Scale bar, $1 \mathrm{~mm}$.

subtypes [1-6]. Our patient noticed muscle atrophy at age 50 . Several LGMD subtypes have recently been identified based upon mutations of certain genes [7-12]; for example, mutations in the calpain 3 and dysferlin genes cause LGMD2A and LGMD2B, respectively.

Recently, some authors have indicated an association between MD and neoplasms [12, 14, 16-20]. MD genes including dystrophin, dysferlin, and calpain 3 act as tumor suppressor genes [21]. Hosur et al. reported that a dystrophin and dysferlin double mutant mice model of human Duchenne Muscular Dystrophy and of LGMD2B each developed rhabdomyosarcoma at an average age of 12 months, with an incidence of $>90 \%$ [14]. Schmidt et al. showed that mutations in MD genes (Dmd, Dysf, Capn3, and LARGE) lead to the spontaneous formation of skeletal muscle-derived malignant tumors in mice, presenting as mixed rhabdomyo-, fibro-, and liposarcomas [12]. The observations of Schmidt et al. suggest that genetically distinct MDs in humans and mice may share a common pathology of cancer-like DNA damage and genomic instability [12].

Liposarcoma is the most common soft tissue sarcoma and accounts for approximately $20 \%$ of all mesenchymal malignancies encountered by practicing surgical pathologists [15]. According to the WHO Classification of Tumors of Soft Tissue and Bone 2013, liposarcomas mainly have four histological subtypes: ALT/WDLS, dedifferentiated liposarcoma, myxoid liposarcoma, and pleomorphic liposarcoma. ALT/WDLS is low to intermediate grade tumors/malignancy and dedifferentiated liposarcoma, myxoid round cell liposarcoma, and pleomorphic liposarcoma are high grade tumors/malignancy. The concept of ALT/WDLS was first reported by Evans et al. in 1979 [22]. They reported that WDLS of subcutaneous tissue and the extremities showed no metastasis, and recommended that the term "well-differentiated liposarcoma" be retained for cases of retroperitoneal tumors because it was difficult in those cases to achieve complete resection and the patients died of local recurrence.

To obtain a definitive diagnosis of ALT based on imaging findings is sometimes difficult because the tumor resembles lipoma. Using standard imaging characteristics (i.e., large size, thick septa, nodules, and nonfatty areas) provides high sensitivity but poor specificity for the diagnosis of ALT due to overdiagnosis of ALT/WDLS from imaging findings [23].

Histologically, ALT is formed by the proliferation of relatively mature adipocytes, consisting of a mixture of mature adipocytes and fibrous connective tissue. The immunohistochemical expression of p16, MDM2, and CDK4 is useful for distinguishing ALT from other lipomatous tumors [24]. In the present case, expression was positive for p16, MDM2, and CDK4, and a definitive diagnosis was thus obtained.

Few reports have examined an association between lipomatous tumors and MD. Yagi et al. reported the association between lipoma and myotonic dystrophy in 2011 [16]. We hypothesize that degenerative changes occurring in adipose tissue during muscle atrophy may cause lipomatous neoplasms; moreover, the mutation of MD-related genes, such as dysferlin, may lead to the proliferation of the tumor or turn into a malignant tumor. To date, to the best of our knowledge, this is the first detailed clinical report to describe an association between ALT and MD, specifically with LGMD2B.

\section{Abbreviations \\ ALT: Atypical lipomatous tumor \\ WDLS: Well-differentiated liposarcoma \\ LGMD: Limb-girdle muscular dystrophy.}

\section{Additional Points}

Availability of Data and Materials. The datasets supporting the conclusions of this article are included within the article.

\section{Ethical Approval}

This study was approved by the Ethics Committee of the Tokushima University Hospital (1942-1).

\section{Consent}

Informed consent was obtained from the patient for publication of this case report. 


\section{Conflicts of Interest}

The authors declare that they have no conflicts of interest, either personal or financial, related to the preparation and publication of this manuscript.

\section{Authors' Contributions}

Ryo Miyagi was involved in the conception and design of the study, interpretation of data, and writing of the manuscript. All authors read and approved the final manuscript.

\section{References}

[1] J. N. Walton and F. J. Nattrass, "On the classification, natural history and treatment of the myopathies," Brain, vol. 77, no. 2, pp. 169-231, 1954.

[2] J. R. W. Yates and A. E. H. Emery, "A population study of adult onset limb-girdle muscular dystrophy," Journal of Medical Genetics, vol. 22, no. 4, pp. 250-257, 1985.

[3] S. H. Laval and K. M. D. Bushby, "Limb-girdle muscular dystrophies-from genetics to molecular pathology," Neuropathology and Applied Neurobiology, vol. 30, no. 2, pp. 91-105, 2004.

[4] H. Gundesli, B. Talim, P. Korkusuz et al., "Mutation in exon If of PLEC, leading to disruption of plectin isoform 1f, causes autosomal-recessive limb-girdle muscular dystrophy," American Journal of Human Genetics, vol. 87, no. 6, pp. 834-841, 2010.

[5] X. Q. Rosales, S. J. Moser, T. Tran et al., "Cardiovascular magnetic resonance of cardiomyopathy in limb girdle muscular dystrophy 2B and 2I," Journal of Cardiovascular Magnetic Resonance, vol. 13, no. 39, 2011.

[6] D. Hicks, A. Sarkozy, N. Muelas et al., "A founder mutation in Anoctamin 5 is a major cause of limb-girdle muscular dystrophy," Brain, vol. 134, no. 1, pp. 171-182, 2011.

[7] E. P. Hoffman, R. H. Brown Jr., and L. M. Kunkel, "Dystrophin: the protein product of the Duchenne muscular dystrophy locus," Cell, vol. 51, no. 6, pp. 919-928, 1987.

[8] R. D. Cohn and K. P. Campbell, "Molecular basis of muscular dystrophies," Muscle and Nerve, vol. 23, no. 10, pp. 1456-1471, 2000.

[9] K. Kobayashi, T. Izawa, M. Kuwamura, and J. Yamate, "Comparative gene expression analysis in the skeletal muscles of dysferlin-defcient SJL/J and A/J mice," Journal of Toxicologic Pathology, vol. 24, no. 1, pp. 49-62, 2011.

[10] H. Sorimachi, N. Toyama-Sorimachi, T. C. Saido et al., "Musclespecific calpain, p94, is degraded by autolysis immediately after translation, resulting in disappearance from muscle," Journal of Biological Chemistry, vol. 268, no. 14, pp. 10593-10605, 1993.

[11] I. Richard, O. Broux, V. Allamand et al., "Mutations in the proteolytic enzyme calpain 3 cause limb-girdle muscular dystrophy type 2A," Cell, vol. 81, no. 1, pp. 27-40, 1995.

[12] W. M. Schmidt, M. H. Uddin, S. Dysek et al., "DNA damage, somatic aneuploidy, and malignant sarcoma susceptibility in muscular dystrophies," PLoS Genetics, vol. 7, no. 4, Article ID e1002042, 2011.

[13] D. Kissling and F. Michot, "Multiple myeloma occurring in association with a preexisting neuromuscular disease (progressive muscular dystrophy): a chance occurrence or a nosological entity?” Acta Haematologica, vol. 72, no. 2, pp. 94-104, 1984.
[14] V. Hosur, A. Kavirayani, J. Riefler et al., "Dystrophin and dysferlin double mutant mice: a novel model for rhabdomyosarcoma," Cancer Genetics, vol. 205, no. 5, pp. 232-241, 2012.

[15] A. P. Dei Tos, "Liposarcoma: new entities and evolving concepts," Annals of Diagnostic Pathology, vol. 4, no. 4, pp. 252-266, 2000.

[16] Y. Yagi, A. Machida, S. Toru et al., "Myotonic dystrophy and lipoma: a new association," Neurological Sciences, vol. 33, no. 6, pp. 1477-1478, 2012.

[17] S. M. Gadalla, M. Lund, R. M. Pfeiffer et al., "Cancer risk among patients with myotonic muscular dystrophy," JAMA, vol. 306, no. 22, pp. 2480-2486, 2011.

[18] A. K. Win, P. G. Perattur, J. S. Pulido, C. M. Pulido, and N. M. Lindor, "Increased cancer risks in myotonic dystrophy," Mayo Clinic Proceedings, vol. 87, no. 2, pp. 130-135, 2012.

[19] G.-M. Lin and Y.-H. Li, "Cancer risk among patients with hereditary muscular dystrophies: a population-based study in Taiwan, 1997-2009," Chinese Journal of Cancer, vol. 33, no. 5, pp. 256-258, 2014.

[20] A. Fanzani, E. Monti, R. Donato, and G. Sorci, "Muscular dystrophies share pathogenetic mechanisms with muscle sarcomas," Trends in Molecular Medicine, vol. 19, no. 9, pp. 546-554, 2013.

[21] Y. Wang, A. Marino-Enriquez, R. R. Bennett et al., "Dystrophin is a tumor suppressor in human cancers with myogenic programs," Nature Genetics, vol. 46, no. 6, pp. 601-606, 2014.

[22] H. L. Evans, E. H. Soule, and R. K. Winkelmann, "Atypical lipoma, atypical intramuscular lipoma, and well differentiated retroperitoneal liposarcoma: a reappraisal of 30 cases formerly classified as well differentiated liposarcoma," Cancer, vol. 43, no. 2, pp. 574-584, 1979.

[23] M. Brisson, T. Kashima, D. Delaney et al., "MRI characteristics of lipoma and atypical lipomatous tumor/well- differentiated liposarcoma: retrospective comparison with histology and MDM2 gene amplification," Skeletal Radiology, vol. 42, no. 5, pp. 635-647, 2013.

[24] K. Thway, R. Flora, C. Shah, D. Olmos, and C. Fisher, "Diagnostic utility of p16, CDK4, and MDM2 as an immunohistochemical panel in distinguishing well-differentiated and dedifferentiated liposarcomas from other adipocytic tumors," The American Journal of Surgical Pathology, vol. 36, no. 3, pp. 462-469, 2012. 


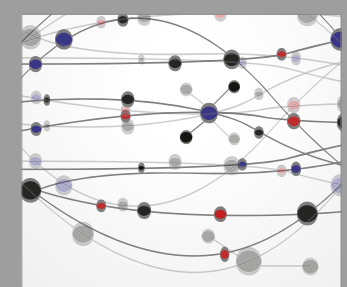

The Scientific World Journal
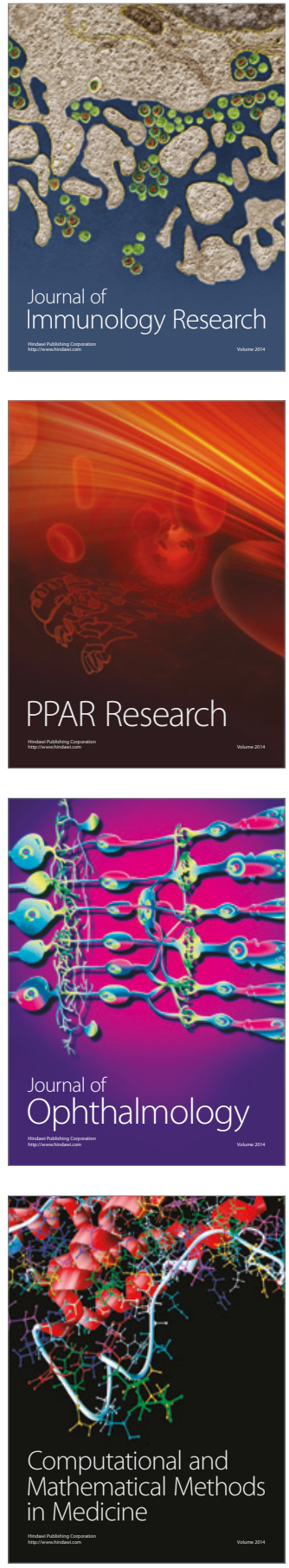

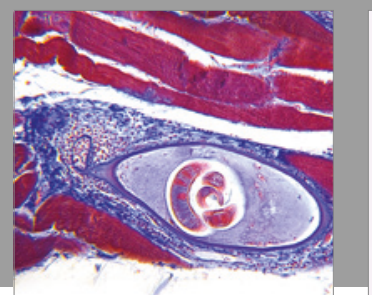

Gastroenterology Research and Practice
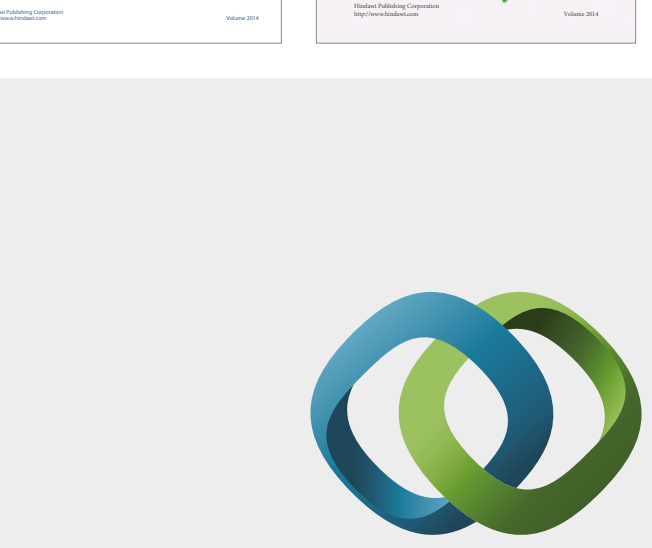

\section{Hindawi}

Submit your manuscripts at

https://www.hindawi.com
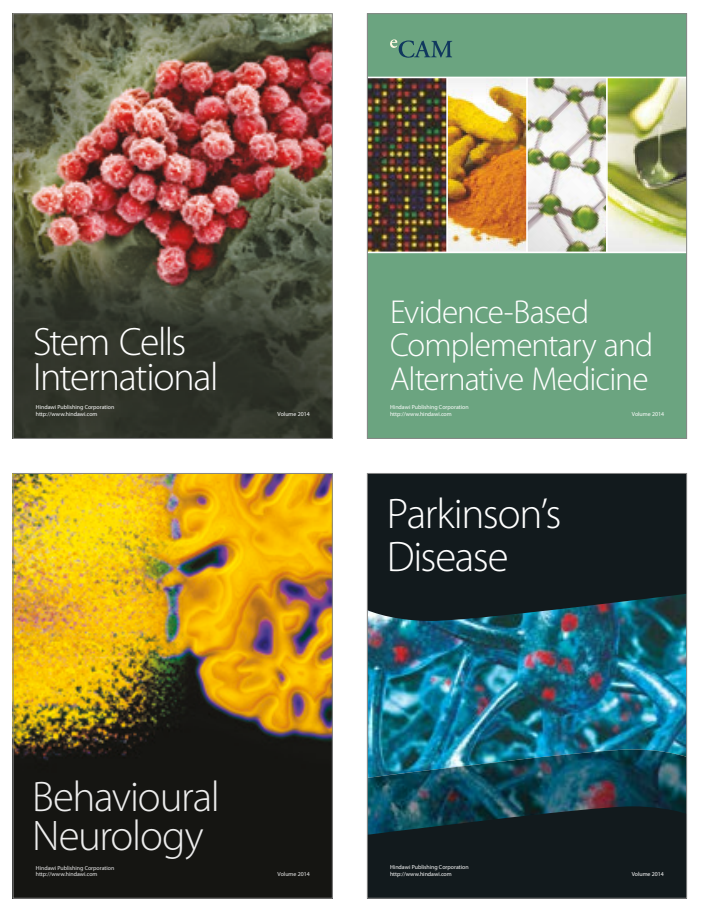
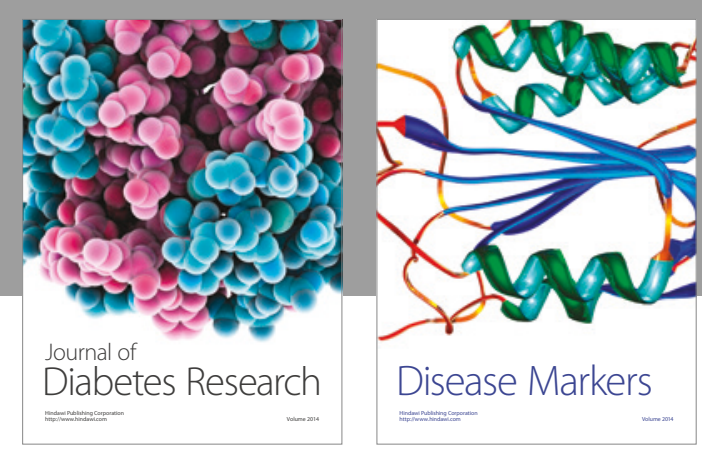

Disease Markers
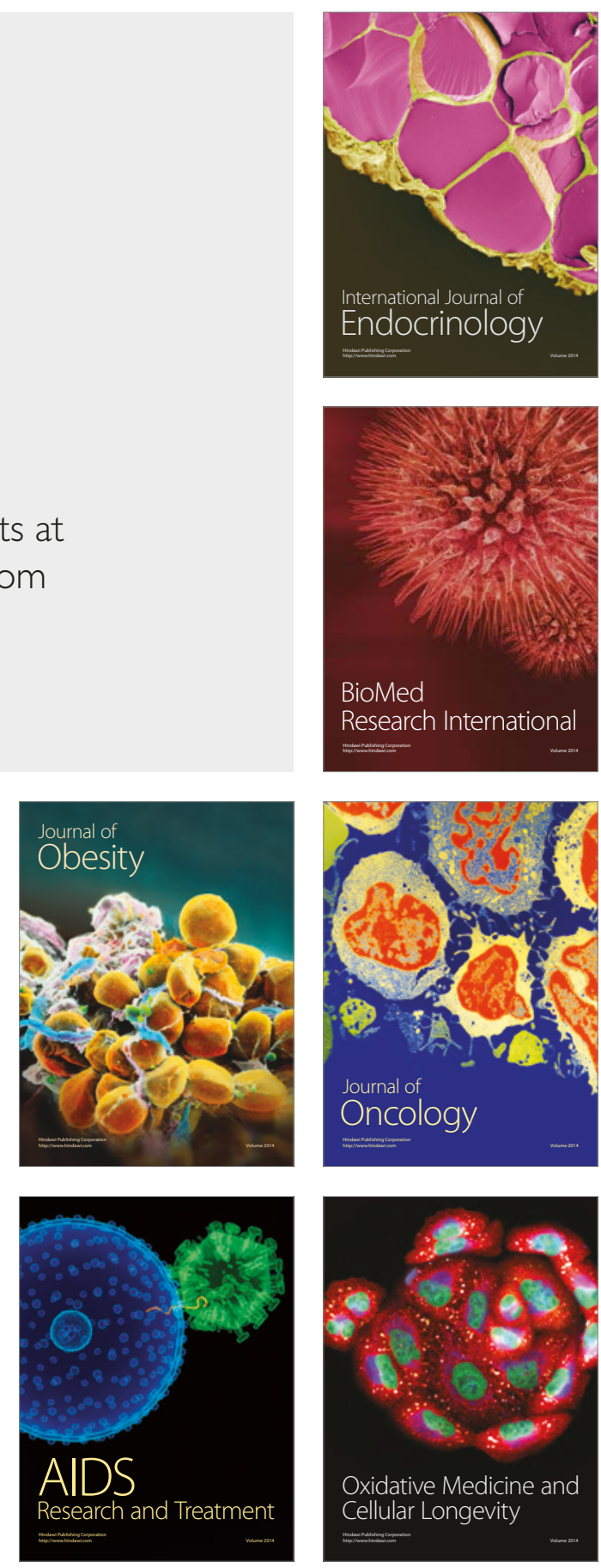